\title{
Artículo
}

\section{Análisis de la producción científica y tecnológica de la industria española en el sector de las Tecnologías de la Información y de las Comunicaciones}

\author{
Por Raquel Rojo e Isabel Gómez
}

\begin{abstract}
Resumen: El estudio presenta una visión general de la producción científica y tecnológica de la industria en el sector de las Tecnologías de la Información y de las Comunicaciones (TIC) durante el período 1996-2000, así como su peso relativo en el conjunto de la producción nacional. A través de indicadores bibliométricos, tomando como unidad de análisis las publicaciones y las patentes, se presentan indicadores a nivel macro-meso por regiones, áreas temáticas y se detectan las empresas que generan los resultados. Se muestra la escasa actividad de $I+D$, el bajo número de patentes, la poca participación de las empresas en publicaciones internacionales, así como la insuficiente interacción entre sector público y privado. La producción científica TIC procede fundamentalmente del sector público (Universidad y CSIC) y la actividad tecnológica está dominada por empresas que patentan principalmente en la Oficina Española. El análisis de las comunidades autónomas españolas refleja una alta concentración en Madrid y Cataluña en publicaciones; sin embargo, Navarra y País Vasco destacan en patentes. Se pone de manifiesto que el sector privado no está rentabilizando los esfuerzos de investigación del sector público para su desarrollo.

Palabras clave: Bibliometría, Producción científica, Actividad tecnológica, Empresas, Patentes, Tecnologías de la información y de las comunicaciones, España, Comunidades autónomas, Innovación.
\end{abstract}

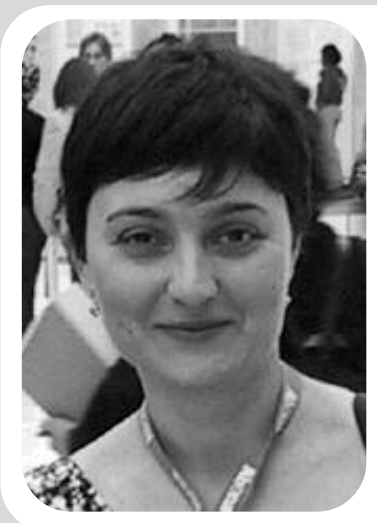

Raquel Rojo es diplomada y licenciada en biblioteconomía y documentación por la Universidad Carlos III de Madrid. En 2001 obtuvo una beca FPI del Ministerio de Ciencia y Tecnología para el desarrollo de su tesis doctoral en el grupo de bibliometría del Cindoc. Dicha tesis, que analiza la producción científica y tecnológica española en el sector de las tecnologías de la información y de las comunicaciones en el período 1991-2000, está actualmente en su fase final de elaboración.

Isabel Gómez, es licenciada en ciencias químicas (UCM), doctora en ciencias (UAM) y profesora de investigación del CSIC. Actualmente es vicedirectora del Centro de Información y Documentación Científica (Cindoc-CSIC) donde dirige el grupo de investigación en bibliometría. Ha participado y dirigido numerosos proyectos de investigación, ha sido autora de más de 50 artículos en revistas científicas de prestigio, así como de diversos capítulos de libros y monografías. Cuenta con una larga experiencia como miembro asesor en diversos comités cien-

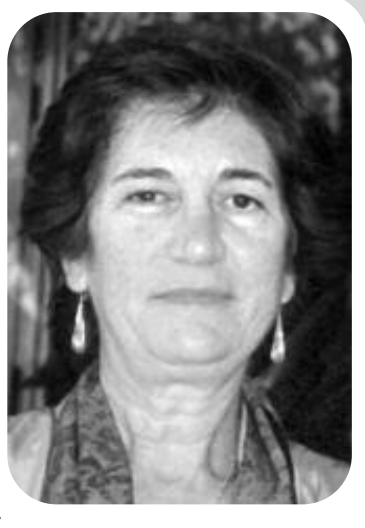
tíficos de revistas, conferencias y organizaciones científicas internacionales del área de la cienciometría.

\section{Title: An analysis of the scientific and technological output of the Spanish Information and} Communication Technologies industry

Abstract: This study presents a general view of the scientific and technological production in the ICT sector of the industry in Spain during the period 1996-2000 and its relative weight in the national production, through bibliometric techniques, with the aim of exploring the character of its outputs, both in terms of publications and patents, focusing the attention on the position and contribution of Spanish ICT scientific and technological capabilities. Indicators at macro-meso level are presented by: geographic region, thematic areas and enterprises. Results about scientific activity in companies show a very short commitment to $R+D$, low number of patents, scarce participation in international publications, as well as insufficient interaction between public and private sectors. Scientific production comes basically from public sector (Universities and CSIC), while the technological activity is located in very few enterprises which patent mainly in the Spanish office. A closer look into Spanish regions reflects a high concentration in Madrid and Catalonia in publications; nevertheless, Navarra and Basque Country stand out in patents. Results reflect that Industry is not taking advantage of the public sector research efforts for its development.

Keywords: Bibliometrics, Scientific production, Technological activity, Enterprises, Patents, Information and Communication Technologies, Spanish regions, Innovation.

Rojo, Raquel; Gómez, Isabel. «Análisis de la producción científica y tecnológica de la industria española en el sector de las tecnologías de la información y de las comunicaciones». En: El profesional de la información, 2006, mayo-junio, v. 15, n. 3, pp. 190-201. 


\section{Introducción}

La industria de las tecnologías de la información y de las comunicaciones (TIC), originada por la convergencia entre las áreas de informática, telecomunicaciones y electrónica, se ha señalado como el principal motor de crecimiento económico en los países desarrollados en los últimos años. En España, este mercado ha generado como media durante el período 1995-2000 el 4,3\% del PIB: 1,7\% procede de las tecnologías de la información y 2,5\% de las telecomunicaciones, según los indicadores de Industria, Innovación y Sociedad de la Información del Ministerio de Industria. Además, se ha definido como un sector intensivo en I+D+I, ya que las empresas TIC presentan resultados superiores a los de la media de la industria española (INE, 1998; Cotec, 2000 y 2001). Ha tenido un crecimiento muy rápido en estos últimos años y se configura como elemento clave para la sociedad de la información.

\section{«La investigación científica e innovación tecnológica del sector privado español es todavía muy deficitaria y se encuentra concentrada en muy pocas empresas»}

Está compuesto por subsectores clasificados por la $O C D E$ como de «alta tecnología» que requieren un continuo esfuerzo en investigación y una sólida base. No sólo es un sector con importancia estratégica para la competitividad del resto de la industria, ya que posibilita el aumento de la productividad gracias a una explotación más eficiente de los factores de producción, sino que también tiene un gran impacto social. Su relevancia política se ha reflejado, por un lado, en su inclusión como objetivo de desarrollo dentro de los planes de investigación a todos los niveles como área científico-tecnológica prioritaria (por ejemplo en los planes nacionales, PN 2000-2003 y 2004-2007) y por otro, se ha tenido en cuenta en los principales informes internacionales sobre indicadores de ciencia y tecnología en los últimos años (ver por ejemplo, NSB, 2002; EC, 2003; OECD, 2003 a, b).

Como consecuencia de este interés socioeconómico, las políticas TIC han ido encaminadas a promover y reforzar la sociedad de la información así como la Nueva Economía en España. Los esfuerzos en este sentido por parte de los diferentes gobiernos se han dirigido tanto a la implantación de infraestructuras y servicios TIC, como a facilitar la penetración de estas tec- nologías en todos los sectores socioeconómicos. Muchas de estas políticas van directamente dirigidas a las pymes para incrementar su competitividad y estimular la inversión privada en investigación e innovación, así como favorecer la creación de nuevas industrias basadas en la tecnología. A nivel nacional y autonómico se han implementado muchos programas e iniciativas para apoyar este sector (Fundación AUNA, 2005; Friedewald et al., 2004).

Para el seguimiento de estas acciones, los gestores a nivel internacional demandan estadísticas que midan el efecto de las políticas e iniciativas TIC que se están promoviendo desde distintos organismos. Así, periódicamente la $O C D E$ y la Comisión Europea a través de Eurostat y EITO ofrecen datos, estadísticas e indicadores del sector $(O E C D, 2000,2002,2003 \mathrm{c}, 2004 \mathrm{a}$, 2004b; EITO, 2001). En este contexto, los indicadores bibliométricos basados en el análisis cuantitativo de las publicaciones científicas y patentes proporcionan una medida de los resultados de la actividad científica y tecnológica con una amplia aceptación y que pueden reflejar conocimientos o tendencias; pero hay que tener en cuenta que las TIC forman un área que comprende una red compleja de resultados de investigación no sólo en forma de publicaciones y patentes.

Asimismo, las patentes constituyen una forma más para las compañías de proteger sus resultados de innovación. Lo que sí es cierto es que las empresas con actividad innovadora patentan al igual que utilizan otras formas de output de la innovación como pueden ser las marcas, modelos de utilidad, diseños y secretos industriales. Estudios bibliométricos que traten explícitamente el sector TIC como un todo son escasos, Tijssen y Van Wijk $(1998,1999)$ presentaron los primeros; y más recientemente las autoras han publicado un artículo acerca del sector TIC en España (Rojo; Gómez, 2006). A nivel más específico podemos encontrar en la literatura trabajos que aplican la bibliometría para examinar un área temática específica de las TIC como la informática (Guan; Ma, 2004) o semiconductores (Tsay; Ma, 2000, 2003). Otros estudios más centrados en la actividad innovadora también aluden al sector (Hicks, et al., 2001; Cotec, 2000, 2001-2003). A nivel regional han aparecido los Observatorios sobre la Sociedad de la Información y la Nueva Economía que hacen el seguimiento del sector (ver por ejemplo IKEI, 2002; OSIN, 2003; Gaptel, 2004) u otros estudios que describen el desequilibrio regional existente en el mismo (Cuadrado-Roura; García-Tabuenca, 2004).

Este análisis propone una visión de la producción científica y tecnológica de la empresa a nivel nacional a través de indicadores bibliométricos basados en el análisis de las publicaciones y las patentes en el sector 


\begin{tabular}{|c|c|c|c|c|}
\hline \begin{tabular}{|c|} 
Bases de \\
datos y \\
tipo de \\
clasificación
\end{tabular} & Informática & $\begin{array}{c}\text { Ingeniería eléctrica y } \\
\text { electrónica }\end{array}$ & Instrumentación & Telecomunicaciones \\
\hline $\begin{array}{c}\text { Inspec } \\
\text { (secciones) }\end{array}$ & Sección "C" y "D" & \begin{tabular}{|l|} 
Sección "B" \\
(excepto B62)
\end{tabular} & Sección "C" y "D" & B62 \\
\hline $\begin{array}{c}\boldsymbol{S C I} \\
\text { (revistas) }\end{array}$ & \begin{tabular}{|l} 
Sistemas de automatización y control \\
Robótica \\
Informática: \\
Inteligencia artificial \\
Cibernética \\
Hardware \\
Sistemas de información \\
Aplic. interdisciplinares \\
Sofware, gráficos y \\
programación \\
Teoría y métodos \\
Informática médica \\
\end{tabular} & Ing. eléctrica y electrónica & Instrumentación & $\begin{array}{l}\text { Control remoto } \\
\text { Telecomunicaciones }\end{array}$ \\
\hline $\begin{array}{c}\text { ICYT } \\
\text { (códigos } \\
\text { Unesco) }\end{array}$ & $\begin{array}{l}\text { Ca }^{a} \text { Ta }^{\mathrm{a}} \text { de ordenadores } \\
(1203 \text { y 3304) }\end{array}$ & $\begin{array}{l}\text { Electromagnetismo (2202) } \\
\text { ca y Ta electrónica ( } 2203 \text { y 3307) } \\
\text { Ing. y Ta eléctricas ( } 3306 \text { ) }\end{array}$ & $\begin{array}{l}\text { Ta instrumentación } \\
(3311)\end{array}$ & $\begin{array}{l}\text { Ta de } \\
\text { telecomunicaciones } \\
(3325) \\
\end{array}$ \\
\hline $\begin{array}{l}\text { Patentes } \\
\text { (códigos } \\
\text { CIP) }\end{array}$ & \begin{tabular}{|l} 
B07C \\
B41J \\
G02F \\
G05F \\
G06C \\
G06F \\
G06G \\
G06K \\
G06M \\
G06T \\
G07B \\
G07C \\
G07D \\
G07F \\
G09G \\
G10L \\
H03K \\
H03L
\end{tabular} & $\begin{array}{l}\mathrm{G} 11 \mathrm{~B} \\
\mathrm{H} 01 \mathrm{~B} \\
\mathrm{H} 01 \mathrm{C} \\
\mathrm{H} 01 \mathrm{~F} \\
\mathrm{H} 01 \mathrm{G} \\
\mathrm{H} 01 \mathrm{H} \\
\mathrm{H} 01 \mathrm{~J} \\
\mathrm{H} 01 \mathrm{~L} \\
\mathrm{H} 01 \mathrm{M} \\
\mathrm{H} 01 \mathrm{R} \\
\mathrm{H} 01 \mathrm{~T} \\
\mathrm{H} 03 \mathrm{~F} \\
\mathrm{H} 03 \mathrm{G} \\
\mathrm{H} 04 \mathrm{H} \\
\mathrm{H} 04 \mathrm{~N} \\
\mathrm{H} 04 \mathrm{R} \\
\mathrm{H} 04 \mathrm{~S}\end{array}$ & $\begin{array}{l}\text { G01B } \\
\text { G01C } \\
\text { G01D } \\
\text { G01F } \\
\text { G01G } \\
\text { G01H } \\
\text { G01J } \\
\text { G01K } \\
\text { G01L } \\
\text { G01M } \\
\text { G01N } \\
\text { G01P } \\
\text { G01R } \\
\text { G01S } \\
\text { G01V } \\
\text { G01W } \\
\text { G02B } \\
\text { G05B } \\
\text { G08G } \\
\text { G09B }\end{array}$ & $\begin{array}{l}\text { G08C } \\
\text { H01P } \\
\text { H01Q } \\
\text { H01S } \\
\text { H03D } \\
\text { HO3H } \\
\text { H03M } \\
\text { H04B } \\
\text { H04J } \\
\text { H04K } \\
\text { H04L } \\
\text { H04M } \\
\text { H04Q }\end{array}$ \\
\hline
\end{tabular}

Tabla 1. Delimitación temática del sector TIC

TIC. Se estudiará en detalle la participación y especialización de las comunidades autónomas teniendo en cuenta no sólo indicadores bibliométricos sino también otros de input del sistema de ciencia-tecnología-empresa.

Algunas de las preguntas de investigación que se plantean son: ¿cuál es la contribución de la empresa española al desarrollo de la investigación TIC?, ¿qué firmas se identifican?, ¿existe alguna especialización en las regiones?, ¿cuáles son las regiones con más orientación TIC? y si es así, ¿cuál es su tipología? Para responder a estas cuestiones se presentan indicadores bibliométricos a nivel macro-meso distribuidos por regiones, áreas temáticas e industrias más productivas. El estudio permitirá identificar las capacidades de investigación y competencias tecnológicas de las diferentes regiones españolas en el sector, lo cual tiene una aplicación en el contexto de la evaluación de las políticas públicas, así como para la práctica estratégi- ca empresarial conocida como «inteligencia competitiva», que busca conocer el entorno con el fin de obtener ventaja frente a la competencia. La bibliometría y, en concreto, el análisis de las patentes pueden esclarecer el comportamiento tecnológico de la competencia.

\section{Metodología}

\section{Obtención de datos}

La producción científica TIC se ha obtenido de distintas bases de datos para el período 1996-2000 en su versión cd-rom. Por un lado, como fuentes internacionales se ha utilizado el Science Citation Index (SCI) elaborada por Thomson-ISI y la base de datos especializada Inspec (Information Services in Physics, Electronics and Computing) que permiten conocer la visibilidad internacional de las publicaciones españolas en esta área. La ventaja de esta última base de datos realizada por el IET (Institution of Engineering and Technology, antes IEE) es que tiene cobertura no sólo de las publicaciones periódicas más punteras, sino que tam- 
bién analiza más revistas nacionales que el $S C I$, además de recoger reuniones científicas que constituyen una fuente importante de difusión de resultados para el sector. La base de datos SCI analiza una selección de las principales revistas internacionales, pero muestra un marcado sesgo hacia títulos de habla inglesa, lo cual supone una limitación importante.

Por otro lado, la publicación nacional de carácter más aplicado se encuentra mejor reflejada en bases de datos locales; por ello, se ha incluido en el estudio la española ICYT, elaborada por el Centro de Información y Documentación Científica (Cindoc-CSIC), que recoge las revistas españolas de ciencia y tecnología y que complementa muy bien a la del $S C I$.

La producción tecnológica española se analiza a través de las bases de datos de patentes de la Oficina Española de Patentes y Marcas (OEPM) y de la Oficina Europea de Patentes (EPO). Se seleccionaron las patentes solicitadas y/o las publicadas durante el período 1996-2000 con solicitante o inventor español, para recoger el mayor número posible de ellas.

El sistema que siguen las oficinas de patentes para incluir los registros en cada base de datos es distinto. En el caso español cuando se solicita la patente ésta permanece en secreto hasta que se publica, y hasta ese momento no se registra en la base de datos correspondiente. El tiempo que puede transcurrir hasta su publicación puede ser de más de 2 años, y de 4 para su concesión, aunque la demora de este proceso es menor en la base de datos europea.

Tan sólo el $10 \%$ de las patentes españolas no se habían aprobado en el período de estudio o, por lo menos, no había datos, sin embargo, en $E P O$ no aparece ninguna patente como concedida. Esto confirma que el proceso de una patente europea conlleva un riguroso examen que no es tan estricto en la oficina española.

\section{Tipo de documentos}

Se han considerado todos, que en las bases de datos interdisciplinares $S C I$ e $I C Y T$ son principalmente artículos de revista; sin embargo, en Inspec el peso relativo de los congresos (conference papers) supone un $70 \%$ de la producción total TIC de la empresa.

\section{Delimitación temática}

El carácter horizontal de las TIC hace que sea un sector de difícil delimitación temática. Sus efectos sobre otras disciplinas originan un alto grado de interdisciplinariedad, a menudo infravalorada. En este estudio nos hemos basado en la definición que hizo la $O C D E$ en 1998 (OCDE, 2000). Así, presentaremos los resultados agrupados en cuatro áreas temáticas: informática, ingeniería eléctrica y electrónica, instrumentación y telecomunicaciones. La división del sector requiere un tratamiento individualizado en cada base de datos debido a las diferentes clasificaciones temáticas y ha sido necesario elaborar una correspondencia entre ellas que permita la comparabilidad de los distintos tipos de outputs.

SCI clasifica los registros asignándolos a las disciplinas científicas de las revistas de publicación. Se ha seleccionado un total de catorce: Control remoto; Informática (incluye: Cibernética, Inteligencia artificial, Hardware, Informática médica, Sistemas de información, Software, Gráficos y programación, Teoría y métodos); Ingeniería eléctrica y electrónica; Instrumentación; Robótica; Sistemas de automatización y control; Telecomunicaciones.

\section{«El bajo número de patentes y la escasa presencia en publicaciones internacionales es un claro reflejo, por una parte, de una capacidad de absorción insuficiente $y$, por otra, de la poca madurez en el mercado internacional de nuestro tejido productivo, compuesto básicamente por pequeñas y medianas empresas»}

Inspec los clasifica en cuatro secciones: A. Física, B. Ingeniería eléctrica y electrónica, C. Informática y control, y D. Tecnologías de la información. Se deslindó el sector TIC seleccionando todas las publicaciones firmadas por una institución española bajo las secciones B, C y D.

ICYT clasifica los documentos según la Nomenclatura internacional de la Unesco. Se eligieron 8 disciplinas a 4 dígitos (ciencia de los ordenadores, tecnología de los ordenadores, electromagnetismo, electrónica, ingeniería y tecnología eléctricas, tecnología electrónica, tecnología de la instrumentación y tecnología de las telecomunicaciones).

En cuanto a las patentes, el sector TIC se ha precisado mediante los siguientes códigos CIP (Clasificación internacional de patentes) a 3 dígitos siguiendo la definición de la OCDE (2004c) y Eurostat (Strack, 2003): G01 excepto G01T, G06, G07, G11; H01, H03 y H04. También se tuvieron en cuenta otros códigos a nivel más detallado de 4 dígitos: B07C, B41J, B41K, G08G, G08C, G09B, G09C, G09G, G10L, G02B6, 
G02F, G03G, G05B y G05F (WIPO, 2000). Los códigos $C I P$ se han agrupado en 4 dominios: informática, ingeniería eléctrica y electrónica, instrumentación y telecomunicaciones siguiendo los criterios de la $O C D E$ (2004c) y la ampliamente aceptada clasificación de la $O S T-I N P I / F h G-I S I$ (Sanz Menéndez; Arias, 1998), recomendada por el manual de la $O C D E$ sobre el uso de las patentes como indicadores de innovación tecnológica (OCDE, 1994).

\section{Tratamiento de los datos}

Los referentes a publicaciones y patentes TIC se transfirieron a una base de datos relacional. La afiliación institucional de los autores fue normalizada mediante una codificación a diferentes niveles que permite identificar la ubicación geográfica y el sector institucional, así como cada una de las empresas (Fernández, et al., 1993). Finalmente, se utilizan programas informáticos desarrollados llevados a cabo en el Cindoc-CSIC para el cálculo de indicadores bibliométricos de ciencia y tecnología.

Además, se han considerado otros indicadores complementarios en el análisis regional relativos a los inputs del Sistema Español de Ciencia y Tecnología, como el gasto en investigación en porcentaje del PIB regional y el número de investigadores en EDP (equivalentes a dedicación plena) por mil activos, como medida del esfuerzo en ciencia de las regiones españolas. Como aproximación a la dedicación tecnológica se ha utilizado el gasto en investigación realizado por las empresas también en porcentaje del PIB regional.

\begin{tabular}{|c|c|c|c|c|c|}
\hline & \multicolumn{3}{|c|}{ Publicaciones } & \multicolumn{2}{|c|}{ Patentes } \\
\hline & Inspec & $S C I$ & ICYT & OEPM & EPO \\
\hline Total TIC & 12.490 & 3.242 & 2.202 & 1.511 & 825 \\
\hline Industria & $\begin{array}{r}369 \\
(3 \%)\end{array}$ & $\begin{array}{r}68 \\
(2 \%)\end{array}$ & $\begin{array}{r}2) \\
(33 \%)\end{array}$ & \begin{tabular}{|r} 
(1) \\
\\
\\
$(46 \%)$
\end{tabular} & $\begin{array}{r}\text { (1) } 354 \\
(43 \%)\end{array}$ \\
\hline Universidad & $\begin{array}{r}(1) \\
10.973\end{array}$ & $\begin{array}{r}(1) \\
2.760 \\
\end{array}$ & $\begin{array}{r}(1) \\
1.240 \\
\end{array}$ & $\begin{array}{r}3) \\
40 \\
\end{array}$ & 33 \\
\hline CSIC & $\begin{array}{r}(2) \\
810 \\
\end{array}$ & $\begin{array}{r}(2) \\
485 \\
\end{array}$ & 101 & 57 & 15 \\
\hline Otros OPI & 101 & 81 & 46 & 6 & 1 \\
\hline Ipsfl & 64 & 35 & 59 & 18 & 2 \\
\hline Particulares & & & & $\begin{array}{r}(2) \\
468 \\
\end{array}$ & $\begin{array}{r}(2) \\
130 \\
\end{array}$ \\
\hline Administración & 41 & 47 & 108 & 7 & 2 \\
\hline
\end{tabular}

Tabla 2. Participación de los sectores institucionales en la producción TIC nacional (1996-2000)

Nota: Se ha detectado un ligero solapamiento entre las diversas bases de datos de publicaciones: 16 documentos Inspec-SCl y 25 Inspec-ICYT. Aproximadamente un $80 \%$ de las patentes europeas (EPO) tienen fecha de prioridad española, es decir que tienen una alta probabilidad de que coincidan con patentes españolas (OEPM) del mismo período.

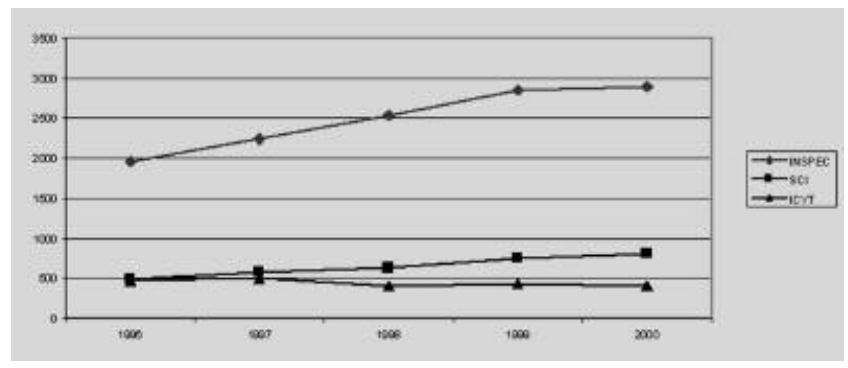

Figura 1. Evolución de las publicaciones TIC en España en todos los sectores (1996-2000)

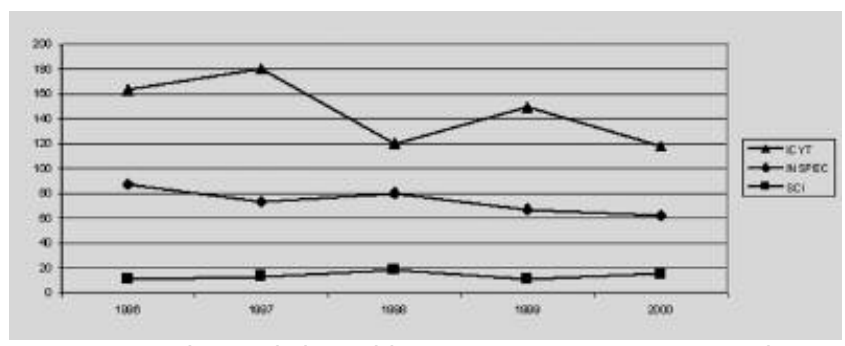

Figura 2. Evolución de las publicaciones TIC en España procedentes de la industria (1996-2000)

\section{Resultados y discusión}

1. Producción científica y tecnológica TIC del sector empresarial (ciencia $v s$ tecnología)

La evolución de las publicaciones en el sector sigue una progresión ascendente en las bases de datos internacionales y ligeramente en descenso en ICYT (figura 1). Se observa una creciente internacionalización de las TIC siguiendo la tendencia que está experimentando la ciencia española (Gómez, I., et al., 2006). Sin embargo, la aportación total de la industria española a publicaciones TIC fue de un $33 \%$ en la base de datos nacional ICYT y muy baja en bases de datos internacionales (3\% y $2 \%$ ) (figura 2), lo cual parece indicar que la investigación más local y de tipo aplicado tiende a publicarse en revistas nacionales.

\section{«Falta eficacia en los mecanismos de interacción entre el sector público y el tejido productivo»}

Si observamos la participación de los distintos sectores institucionales, el público es el principal actor de la investigación TIC, principalmente la universidad con más de un $85 \%$ de las publicaciones internacionales. La escasa presencia de las empresas en éstas va acompañada por una también limitada relación con el sector público que se refleja en la poca colaboración que se da entre ambos sectores en publicaciones cien- 


\begin{tabular}{|c|c|c|c|c|c|c|c|c|}
\hline \multirow[b]{3}{*}{\begin{tabular}{|l|}
$\begin{array}{l}\text { Comunidades } \\
\text { Autónomas }\end{array}$ \\
\end{tabular}} & \multicolumn{5}{|c|}{ Output 1996-2000 } & \multicolumn{3}{|c|}{ Input referido a año 2000} \\
\hline & \multicolumn{3}{|c|}{$\begin{array}{l}\begin{array}{l}\text { Publicaciones } / 10^{6} \\
\text { habs. }\end{array} \\
\end{array}$} & \multicolumn{2}{|c|}{ Patentes $/ 10^{6}$ habs. } & \multirow{2}{*}{\begin{tabular}{|l} 
Investigadores \\
en empresas \\
EJC/ \\
$10^{3}$ activos \\
\end{tabular}} & \multicolumn{2}{|c|}{ Intensidad en I+D } \\
\hline & INSPEC & $S C I$ & ICYT & $O E P M$ & EPO & & \begin{tabular}{|l|}
$\begin{array}{l}\text { Gasto I+D/ } \\
\% \text { PIB regional }\end{array}$ \\
\end{tabular} & $\begin{array}{l}\text { Gasto I+D empresas/ } \\
\% \text { PIB regional }\end{array}$ \\
\hline Andalucía & 1,6 & & 2,0 & 2,2 & 0,7 & 0,21 & 0,67 & 0,22 \\
\hline Aragón & & & 5,0 & 16,0 & 5,9 & 0,55 & 0,71 & 0,40 \\
\hline Asturias & & & 10,2 & 3,7 & & 0,24 & 0,81 & 0,41 \\
\hline Baleares & & & & & & 0,01 & 0,25 & 0,03 \\
\hline C. Valenciana & 4,9 & & 2,4 & 9,2 & 6,3 & 0,40 & 0,74 & 0,31 \\
\hline Canarias & & & & & & 0,03 & 0,49 & 0,10 \\
\hline Cantabria & & & & & & 0,07 & 0,47 & 0,11 \\
\hline Castilla y León & & & & 2,4 & & 0,23 & 0,64 & 0,27 \\
\hline Castilla-La Mancha & & & & & & 0,25 & 0,57 & 0,36 \\
\hline Cataluña & 5,7 & 1,3 & 21,7 & 30,0 & 22,2 & 0,81 & 1,11 & 0,75 \\
\hline Extremadura & & & & & & 0,12 & 0,54 & 0,14 \\
\hline Galicia & & & 4,4 & 2,9 & & 0,14 & 0,63 & 0,21 \\
\hline La Rioja & & & & & & 0,22 & 0,61 & 0,36 \\
\hline Madrid & 51,5 & 7,3 & 90,3 & 55,3 & 22,1 & 1,29 & 1,67 & 0,91 \\
\hline Murcia & 3,5 & & & & & 0,12 & 0,74 & 0,31 \\
\hline Navarra & & & & 97,5 & 71,7 & 0,72 & 0,92 & 0,60 \\
\hline País Vasco & 7,6 & 5,2 & 21,0 & 32,9 & 10,0 & 1,34 & 1,20 & 0,91 \\
\hline España & 9,1 & 1,7 & 18,0 & 17,2 & 8,7 & 0,52 & 0,94 & 0,50 \\
\hline
\end{tabular}

Tabla 3. Indicadores regionales TIC del sector industrial (valores relativos)

Fuente: datos de input para el año 2000 procedentes de la estadística sobre actividades de l+D del INE. http://www.ine.es/inebase/

tíficas. En cuanto a la producción tecnológica, la empresa es el primer sector en patentes, con porcentajes muy similares tanto en españolas (46\% del total nacional TIC) como en europeas (43\%).

La investigación española TIC del sector empresarial es fundamentalmente de carácter nacional y queda mejor reflejada en publicaciones españolas (33\% en ICYT); y su actividad tecnológica en las patentes (más de un $40 \%$ ). Los resultados sugieren que, para el estudio del comportamiento de la empresa en un sector tecnológico que produce principalmente un tipo de investigación orientada, sea menos adecuado el uso de bases de datos de publicaciones internacionales, pero es útil su uso en cuanto a que es un indicador de la ciencia básica interna en la empresa y de visibilidad más internacional (figura 3).

Respecto a cómo las empresas difunden sus resultados de investigación, se observa una pauta interesante: mientras que en las bases de datos interdisciplinares $S C I$ e ICYT la comunicación de los resultados se da en forma de artículo de revista en más de un $95 \%$, en
Inspec se ha observado una importante presencia de las presentaciones a congresos que suponen el $70 \%$ de la producción industrial. Esto es un reflejo de la importancia que tienen las reuniones científicas y eventos profesionales para esta comunidad científica (Rojo; Gómez, 2006), hecho que se ha puesto de manifiesto como una de las fuentes principales para la innovación que han señalado las propias empresas en la tercera encuesta comunitaria sobre innovación (CIS3) publicada por Eurostat (EC, 2005).

\section{Dominios temáticos}

Considerando las cuatro grandes áreas mencionadas, las empresas muestran una tendencia hacia la ingeniería eléctrica y electrónica en publicaciones y patentes europeas, mientras que en patentes registradas en la $O E P M$ se orientan a informática. En todos los casos predomina la producción nacional sobre la internacional, excepto en las publicaciones de ingeniería eléctrica y electrónica e informática, en las que coincide Inspec con ICYT (figura 4). 


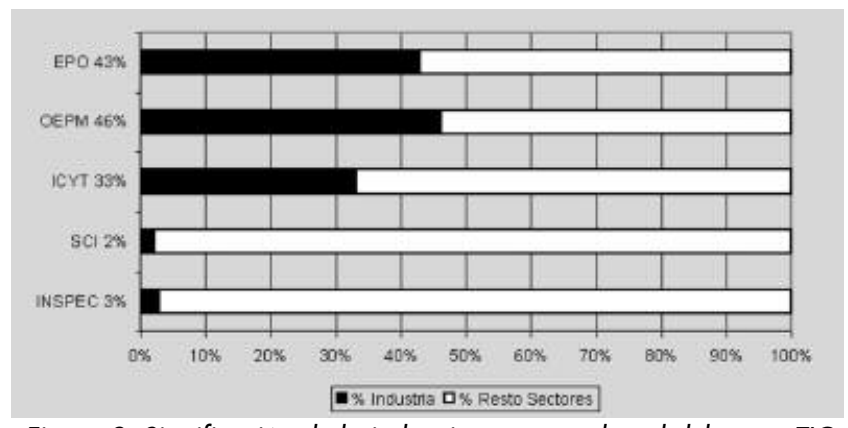

Figura 3. Significación de la industria respecto al total del sector TIC en España en porcentaje (1996-2000)

\section{Regionalización de la producción TIC em- presarial}

Ya que las cifras absolutas de la industria en el sector TIC son bajas en cuanto al output científico y tecnológico, en la tabla 3 sólo se indican los valores relativos de aquellas comunidades autónomas con más de tres publicaciones o patentes en cada una de las fuentes de datos analizadas. Los indicadores se han normalizado de acuerdo con su población, lo que permite identificar regiones que, aunque más pequeñas, puedan mostrar una actividad importante.

\section{«El Sistema Español de Innovación sigue siendo una asignatura pendiente en el proceso de convergencia con Europa»}

En números absolutos se observa una gran concentración en Madrid en publicaciones (73\% en Inspec, $56 \%$ en SCI y $64 \%$ en ICYT). Sin embargo, en patentes la concentración se reparte entre Madrid y Cataluña, pues ambas acaparan el 68\% de españolas y el $72 \%$ de las europeas del sector TIC. En valores relativos, Navarra y País Vasco destacan por su visibilidad sobre todo en patentes, muy por encima de la media española. Estas regiones se encuentran también por encima de la media nacional tanto en los indicadores relativos al esfuerzo en I+D e Innovación, como en el gasto en alta tecnología, lo que puede tomarse como una medida de su competitividad. Las regiones españolas objetivo 1 concentran un gasto mayor en actividades industriales de baja intensidad tecnológica (Acosta; Coronado, 2003) y que además muestran una menor capacidad de absorción de los fondos públicos de investigación en comparación con las regiones más desarrolladas (Cuadrado-Roura; García-Tabuenca, 2004) -es lo que se conoce como «paradoja de la innovación regional» (Landabaso, 2000)-.

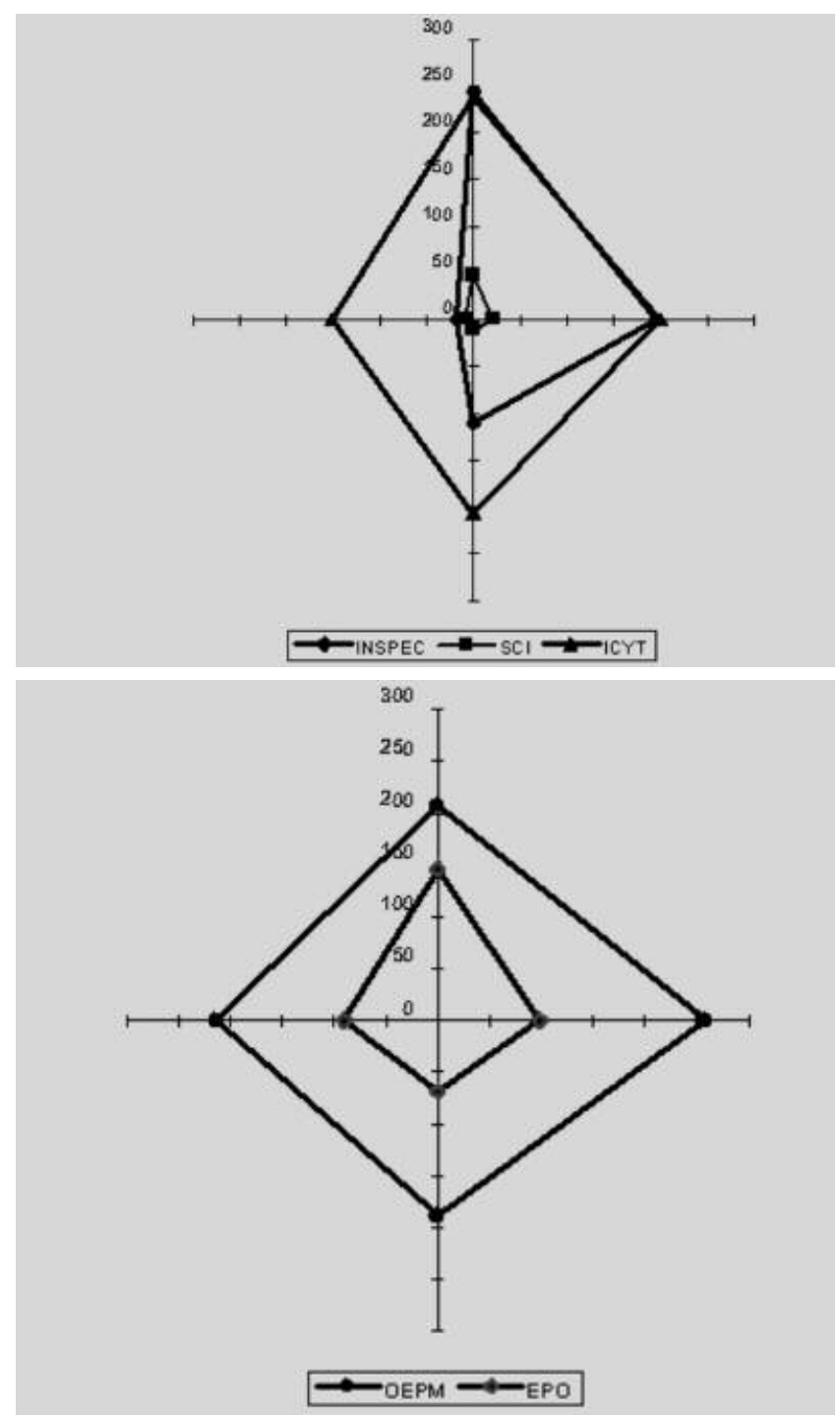

Figura 4. Publicaciones y patentes internacionales y nacionales TIC de la industria española por áreas científico-tecnológicas (1996-2000)

\section{Concentración y especialización de la activi- dad en TIC}

Madrid es la región líder en todos los indicadores de resultados de la actividad científica y tecnológica. Se aprecia cierta especialización en telecomunicaciones, tanto en publicaciones españolas como en patentes. Sin embargo, en las internacionales muestra mayores valores en ingeniería eléctrica y electrónica e informática (tabla 4). Cataluña presenta una mayor actividad tecnológica y las empresas muestran una especialización en patentes en ingeniería eléctrica y electrónica. Otras comunidades muy orientadas a tecnología TIC son Navarra, especializada en patentes de informática y País Vasco, que se inclina tanto por la informática como por la instrumentación.

Los principales factores que hay detrás de estos resultados los encontramos en las políticas regionales, así como en la estructura productiva de estas regiones, sin perder de vista además las infraestructuras para la innovación: estas comunidades poseen parques tecno- 


\begin{tabular}{|l|r|r|r|r|r|r|r|r|r|r|r|r|}
\cline { 2 - 13 } \multicolumn{1}{c|}{} & \multicolumn{9}{c|}{ Publicaciones } \\
\hline Comunidades & \multicolumn{3}{c|}{ Inspec } & \multicolumn{3}{c|}{ SCI } & \multicolumn{3}{|c|}{ ICYT } \\
\cline { 2 - 13 } & A & B & C & D & A & B & C & D & A & B & C & D \\
\hline Andalucía & 3 & 10 & 2 & 3 & 1 & 3 & & 1 & 5 & 4 & 4 & 2 \\
\hline Aragón & 1 & 1 & & & & 2 & & & 1 & 5 & & \\
\hline Asturias & 1 & 1 & & & & & & & 4 & 6 & & 1 \\
\hline Baleares & & & & & & 2 & 2 & & & 2 & 1 & \\
\hline C. Valenciana & 3 & 19 & 1 & & & 2 & & 1 & 2 & 7 & 1 & 2 \\
\hline Canarias & & & & & & & & & 1 & & & 1 \\
\hline Cantabria & 1 & 3 & & & & 1 & & 1 & & 2 & 2 & 1 \\
\hline Castilla y León & & & & & & & & & & 2 & 1 & \\
\hline Castilla-La & & 1 & & & & & & & 1 & & & \\
Mancha & & 1 & & & & & & & & & & \\
\hline Cataluña & 23 & 21 & 8 & 2 & 4 & 7 & 4 & 2 & 35 & 59 & 11 & 42 \\
\hline Extremadura & & & & & & 1 & & 1 & & 1 & & \\
\hline Galicia & & & & & & 2 & 1 & 1 & & 10 & 1 & 2 \\
\hline La Rioja & & & & & & & & & & & & \\
\hline Madrid & 147 & 173 & 93 & 13 & 11 & 29 & 6 & 3 & 128 & 124 & 181 & 78 \\
\hline Murcia & 4 & 2 & 1 & & 3 & & & & & & & 1 \\
\hline Navarra & & & & & & 2 & & 2 & & & & 1 \\
\hline País Vasco & 10 & 8 & 3 & & 4 & 11 & 1 & 2 & 17 & 13 & 4 & 17 \\
\hline España TIC & & & 369 & & & $\mathbf{6 8}$ & & & $\mathbf{7 3 0}$ \\
\hline
\end{tabular}

\begin{tabular}{|r|r|r|r|r|r|r|r|}
\hline \multicolumn{7}{|c|}{ Patentes } \\
\hline OEPM & B & C & D & A & B & C & D \\
\hline 8 & 2 & 2 & 5 & 3 & & & 2 \\
\hline 6 & 7 & 4 & 4 & 1 & 4 & 1 & 1 \\
\hline 1 & 2 & & 1 & & & & \\
\hline & & & 1 & & & & \\
\hline 14 & 11 & 2 & 16 & 4 & 12 & 5 & 6 \\
\hline & & & & & & & \\
\hline & & & & & & & \\
\hline 2 & 2 & 1 & 2 & 1 & & 1 & \\
\hline & 1 & & & & 1 & & 1 \\
\hline 42 & 87 & 15 & 55 & 14 & 81 & 8 & 37 \\
\hline 1 & & & 1 & & & & \\
\hline 2 & 3 & & 4 & & 2 & & \\
\hline & & & 2 & & & & \\
\hline 88 & 61 & 115 & 67 & 31 & 28 & 41 & 30 \\
\hline & & & 2 & & & & \\
\hline 40 & 9 & & 9 & 32 & 6 & 1 & 2 \\
\hline 21 & 11 & 15 & 30 & 5 & 9 & 1 & 6 \\
\hline & & $\mathbf{6 9 7}$ & & & $\mathbf{3 5 4}$ \\
\hline
\end{tabular}

Tabla 4. Especialización TIC de las empresas de las diferentes regiones españolas (valores absolutos).

A: Informática; B: Ingeniería eléctrica y electrónica; C: Telecomunicaciones; D: Instrumentación.

lógicos especializados en TIC según datos de la Asociación de Parques Tecnológicos de España (APTE). Los resultados coinciden con las observaciones de otros autores: estas comunidades con especialización TIC son también las más orientadas a tecnología, poseen un tejido industrial activo y apuestan por la innovación como factor de liderazgo (Gómez, I. et al., 2005).

\section{5. ¿Qué empresas tienen actividad en TIC?}

Las más productivas tanto en publicaciones como en patentes se presentan en las figuras 5 y 6 . Las ma-

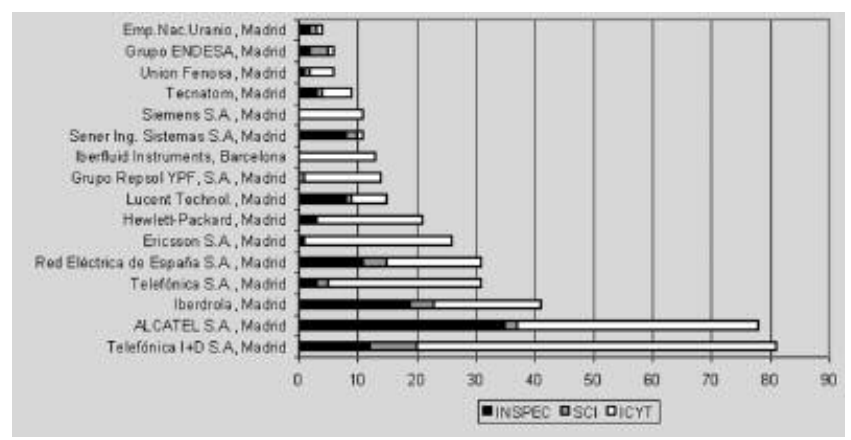

Figura 5. Empresas con más producción en publicaciones (>5) drileñas son las que más publican, pero la presencia de otras comunidades en patentes es notable. Telefónica y Alcatel, que centran su actividad en telecomunicaciones, son las únicas empresas con output en las 5 fuentes de información y son las compañías más productivas en Madrid de acuerdo con la especialización temática de esta comunidad (tabla 4). Es destacable el papel de Telefónica como una de las empresas líderes en TIC, siendo la única compañía española que ha aparecido en el ranking de las 50 empresas europeas en inversión en I+D del mundo en 2004 (EC, 2005).

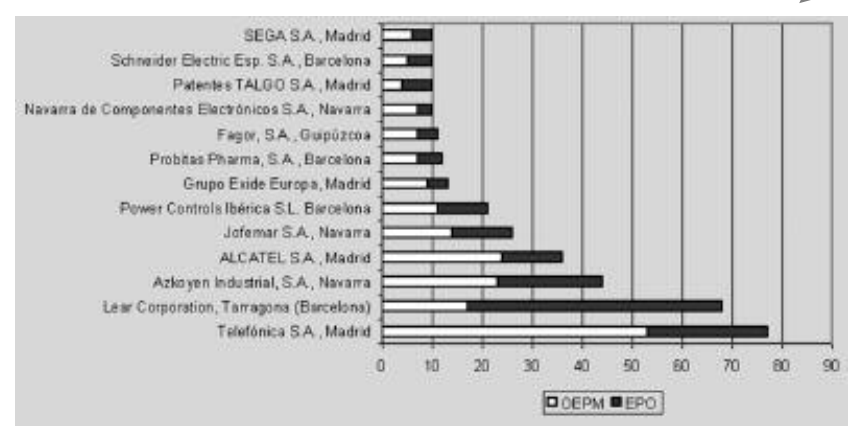

Figura 6. Empresas con más producción en patentes $(>10)$ 
Las empresas catalanas líderes en patentes pertenecen al subsector de los componentes eléctricos del automóvil, es el caso de Lear. Navarra tiene tres entre las más activas de España: Azkoyen y Jofemar dedicadas a la maquinaria de venta automática y Navarra de Componentes Electrónicos especializada en materiales eléctricos y electrónicos.

\section{Conclusiones}

La investigación científica e innovación tecnológica del sector privado español en las TIC es todavía muy deficitaria y además se encuentra concentrada en muy pocas empresas. La investigación básica TIC procede del sector público, los grupos de investigación proceden de la universidad y del CSIC (Rojo; Gómez, 2006). La actividad tecnológica se refleja en las patentes sobre todo de la empresa (más del $40 \%$ ) principalmente registradas en la oficina española aunque la actividad patentadora es muy baja. A pesar de que las solicitudes de patentes a la oficina europea $(E P O)$ han crecido más rápidamente que la media de solicitudes en todos los demás sectores (durante la década de los 90 aumentaron un $8,9 \%$ anual cuando todas las solicitudes de patentes lo hicieron a un 6,7\%) Europa no está especializada en patentes TIC, mientras que Japón y Estados Unidos sí lo están según el índice de especialización que ha publicado la $O C D E$ en un informe reciente. Entre los países de la $O C D E$ especializados en patentes TIC aparecen Finlandia, Islandia, Corea y los Países Bajos. Este indicador va acompañado en estos países de un gasto I+D de las empresas TIC también alto $(O E C D, 2003 b)$.

\section{«La investigación del sector empresarial es de carácter nacional y queda mejor reflejada en publicaciones españolas. No obstante, se observa una creciente internacionalización TIC siguiendo la tendencia que está experimentando la ciencia española»}

Este sector, a pesar de ser altamente tecnológico, hereda las características de la situación de la investigación española, con una mayoría de regiones que presentan mejor resultado científico que tecnológico y muy influenciado por el conocido fenómeno de la «paradoja europea». En este sentido, hay autores que hablan de «paradoja regional»-citada antes- para denotar que las regiones no están utilizando la ciencia bási- ca para desarrollar su tejido empresarial, es decir no tienen capacidad de convertir en valor económico sus esfuerzos en investigación e innovación (Coronado; Acosta, 2005).

El desequilibrio estructural que muestra el sistema de ciencia y tecnología a nivel europeo viene dado por la poca participación empresarial, exceptuando algunos países. Para paliar esta situación, Europa pretende aumentar la inversión en I+D en porcentaje del PIB hasta un $3 \%$ y que un $66 \%$ de la financiación de la I+D provenga de la empresa. Estos objetivos deben alcanzarse en 2010 y fueron enunciados por primera vez en la Estrategia de Lisboa (2000) y retomados después en el Consejo Europeo que tuvo lugar en Barcelona (2002).

En 1990, Cohen y Levinthal introdujeron el concepto de capacidad de absorción de la empresa, que hace referencia a su habilidad para identificar, asimilar y explotar el conocimiento proveniente de fuentes externas dirigido a crear y sostener ventajas competitivas (Cohen; Levinthal, 1990). Uno de los efectos de la innovación en la empresa es que suele traer consigo cambios estratégicos en su estructura organizativa que, a su vez, favorece la misma (Pulido, 2005); de hecho, en España el 52\% de las empresas innovadoras implantaron cambios en su estructura organizativa según datos de la última encuesta comunitaria CIS3. Estas modificaciones pueden ser determinantes para favorecer esta capacidad de absorción en nuestras empresas, que se impone necesaria, y que puede ayudar a corregir la actual situación de retraso de España respecto de los países de referencia en relación con indicadores de ciencia, tecnología e innovación y posibilitar así un sector TIC realmente competitivo en el ámbito internacional. Si las empresas del sector a nivel europeo saben aprovechar los recursos propios, en este caso la investigación básica, tanto para comercializar como para incorporar tecnología, la capacidad de innovación de nuestras empresas se verá aumentada. España presenta uno de los porcentajes más bajos de empresas innovadoras, consecuencia de una estructura industrial basada en actividades de bajo contenido tecnológico, evidencia ya constatada en otros estudios (Fernández de Lucio, et al., 2003; Navarro, 2002).

El bajo número de patentes y la escasa presencia en publicaciones internacionales es un claro reflejo, por una parte, de una capacidad de absorción insuficiente y, por otra, de la poca madurez en el mercado internacional de nuestro tejido productivo, exceptuando algunos casos. Una situación avalada por el continuo saldo negativo de nuestra balanza que nos señala como un país importador de tecnología y que debe realizar un mayor esfuerzo para registrar no sólo patentes domésticas sino también internacionales vía europea o 
PCT (Patent Cooperation Treaty, de la OMPI). Mejorar el aprovechamiento de los resultados de la I+D e innovación es una medida básica que debe estar en la política industrial del sector TIC y que ya se contempla en el Plan Avanza (Ministerio de Industria, Turismo y Comercio, 2005). Este plan, que forma parte del Programa Ingenio 2010 que ha lanzado a finales de año el gobierno para cumplir con la Estrategia de Lisboa, contempla una serie de medidas para el período 2006-2010 para las TIC que presumiblemente van a tener un gran impacto en su desarrollo.

\section{«Existe una alta concentración en Madrid y Cataluña en todos los indicadores presentados, pero la presencia de otras comunidades como Navarra y País Vasco en patentes TIC es notable»}

Las empresas españolas deben incorporar a su estrategia empresarial las actividades de I+D de cara a la innovación en su propio beneficio y mantener un alto grado de compromiso en esta materia pasando de ser un actor secundario a ser el principal agente de ejecución del gasto en I+D con porcentajes próximos a las 2/3 partes del total, como en los países más desarrollados. Desde el punto de vista de la financiación del gasto en I+D, las empresas españolas y europeas (financian el 47 y $56 \%$ respectivamente) están muy lejos de los niveles satisfactorios que revela un sistema maduro de investigación y más desarrollado (más del 60\%); países como Estados Unidos, Japón, Alemania, Bélgica, Finlandia, Suecia y Corea son ejemplos, en estos países las empresas constituyen un componente fundamental de sus Sistemas. En España, desde el punto de vista de la ejecución del gasto en I+D, la empresa es su principal ejecutor, pero los resultados de la I+D empresarial son muy pobres y sólo emplea al $30 \%$ de investigadores. El sector público también debe contribuir a subsanar esta y otras fallas del Sistema Español de Ciencia y Tecnología, como la falta de eficacia de los mecanismos de interacción entre el sector público y el tejido productivo en lo referente a I+D. En este sentido, fortalecer la relación entre los diversos agentes del sistema, buscar la complementariedad entre ellos y promover actuaciones de carácter vertical, y revisar los mecanismos de transferencia de tecnología, están entre los objetivos de la política científica española para converger. Las organizaciones interfaz, que cumplen estas funciones de interrelación, tienen un papel favorecedor tanto de los flujos de transferencia de conocimiento y tecnología como para los procesos de innovación. Su número, distribución y gestión eficaz son claves para los sistemas nacionales y regionales de innovación. Entre estas entidades se incluyen Fundaciones Universidad-Empresa, Oficinas de Transferencia de Resultados de Investigación, consultores y/o institutos tecnológicos, parques tecnológicos, entidades de capital riesgo, entre otras. Las políticas de innovación deben prestar especial atención a la creación de redes de cooperación con las empresas y centros tecnológicos: no sólo mejorarán la capacidad de absorción y de innovación de éstas sino que conducirán a la creación de empresas basadas en ciencia o conocimiento (Castro; Fernández de Lucio, 2006).

La utilización de diferentes fuentes pone de manifiesto que el tipo de investigación TIC que está publicando la empresa es de naturaleza aplicada (más presencia en publicaciones españolas) especialmente en telecomunicaciones e informática, por lo que cabe preguntarse si ello tiene alguna relación, quizá, con la difusión de una patente previa y observar más detenidamente esa relación. En cambio, la ingeniería eléctrica y electrónica de tipo más básico se refleja mejor en publicaciones internacionales.

A nivel regional se observa una alta concentración en Madrid y Cataluña en todos los indicadores presentados referidos a la investigación e innovación TIC, siendo Madrid la región líder en cuanto a actividad científica y tecnológica junto con una importante presencia TIC de Navarra y País Vasco. Zitt y otros autores (2003) han señalado que la concentración del resultado científico y tecnológico en pocas regiones, característica de España, Francia, Suecia, Dinamarca y los Países Bajos hace que posean un alto potencial para generar efectos spillover (la concentración de recursos produce más impactos positivos de los esperados). De hecho se ha comprobado que un entorno científico favorable en la región es un determinante para que las compañías situadas en ella hagan mayor uso de la ciencia en la tecnología que patentan. En España, los flujos entre ciencia y tecnología medidos en términos de citas en patentes empresariales a publicaciones científicas se centralizan en tres comunidades autónomas: Cataluña, Madrid y Navarra (Coronado; Acosta, 2005).

El resto de comunidades no muestran una actividad relevante en TIC en nuestro estudio. En un informe elaborado por las cámaras de comercio, industria y navegación de España sobre la incorporación de las TIC en las comunidades autónomas (y cuyos resultados figuran en su Informe Económico Anual del año 2000) identifica comunidades con valores más positivos en otro tipo de indicadores relacionados con la presencia de las TIC (número de empresas, recursos 
humanos, mercado TIC) como La Rioja; o con su uso como Baleares y Canarias.

No se puede hablar de especialización en las comunidades autónomas en lo que se refiere al output científico y tecnológico empresarial porque las cifras son muy bajas, exceptuando el caso de la empresas de Madrid en el subsector de las telecomunicaciones. Otros autores han observado diferencias y similitudes entre las regiones españolas en cuanto a si sus actividades de I+D están más orientadas a ciencia o si por el contrario presentan un mejor resultado tecnológico como Cataluña, Navarra y País Vasco (Gómez, I. et al., 2005). Entre los factores explicativos de estas diferencias podemos enumerar los planes regionales de investigación, la política industrial y tecnológica de las diferentes comunidades autónomas junto con sus características socioeconómicas y su estructura productiva.

\section{«El sector privado no está rentabilizando los esfuerzos de investigación del sector público para su desarrollo. Las empresas españolas deben incorporar a su estrategia empresarial las actividades de I+D de cara a la innovación»}

En cuanto a los indicadores de penetración TIC en las comunidades observamos serias divergencias entre ellas. En este sentido, el Plan Avanza busca reducir esas diferencias, facilitando la implantación de las TIC en el tejido productivo para así contribuir al éxito de un modelo de crecimiento económico basado en el incremento de la competitividad y la productividad. El entramado empresarial en España está compuesto en más de un $94 \%$ por pymes cuyo nivel de incorporación de las TIC se encuentra muy por debajo del que presentan las grandes empresas, y es especialmente preocupante en la pequeña empresa (menos de 10 empleados). El Plan busca la consecución de un conjunto de objetivos estratégicos en línea con la agenda de Lisboa, que persigue la convergencia con los países europeos más avanzados, teniendo en cuenta que ese proceso también ha de efectuarse a nivel nacional entre las comunidades autónomas. El Plan Avanza pretende conseguir que el volumen de la actividad económica relacionada con las TIC se acerque al 7\% del PIB en 2010, para lo que será necesario un esfuerzo conjunto por parte del sector privado y las distintas administraciones.
El sistema español de innovación sigue siendo una asignatura pendiente en el proceso de convergencia con Europa. En el año 2000 los gastos en I+D de las empresas suponían el 0,50\% del PIB frente a una media de $1,22 \%$ en la UE-15 y el $1,56 \%$ en la $O C D E$. España se encuentra a la cola, sólo por delante de Grecia y Portugal, y reducir la acusada brecha tecnológica que nos separa del resto de la UE no sólo requiere una actuación pública en términos de aumentar el gasto en $\mathrm{I}+\mathrm{D}$.

El esfuerzo realizado desde la aprobación del $I$ Plan Nacional en 1988 ha fortalecido notablemente el Sistema Español de Ciencia-Tecnología-Empresa pero todavía acumulamos un retraso considerable en los indicadores que lo describen respecto a los países de nuestro entorno científico ya que, en la práctica, la existencia de la idea de esta política como una estrategia global es muy reciente y tuvo lugar a finales de la década de los 90 con la aprobación del IV Plan Nacional 2000-2003.

El Plan Nacional de I+D, el Plan de Fomento Empresarial y el Plan Avanza, son algunas medidas que van a resultar decisivas, por un lado para el impulso y desarrollo del sector TIC, y por otro para que el sistema de innovación se integre en la cultura empresarial como un elemento esencial del tejido industrial en España.

La investigación e innovación en las TIC, así como su aplicación, generan impactos de tipo económico de doble naturaleza, por un lado, en sentido vertical, en las ramas de actividad propias del sector, y por otro lado, en sentido horizontal en el resto de actividades productivas de bienes y servicios. Según estimaciones de la $O C D E$, hasta un $40 \%$ de las mejoras de la productividad en los últimos años en los Estados Unidos se han originado gracias a la producción y la implantación de las TIC (Pulido, 2005).

\section{Agradecimientos}

Al Ministerio de Ciencia y Tecnología por la financiación de la investigación mediante una beca predoctoral FPI vinculada al proyecto SEC2000-1312. Parte de los resultados se presentaron en las I Jornadas de indicadores para la evaluación de la ciencia y la tecnología organizadas por el Cindoc-CSIC. Un resumen del póster está disponible en la web:

http://www.cindoc.csic.es/info/fesabid/24.htm

\section{Bibliografía}

Acosta, M.; Coronado, D. «Science-technology flows in Spanish regions. An analysis of scientific citations in patents». En: Research policy, 2003, December, v. 32, n. 10, pp. 1.783-1.803.

Castro, E.; Fernández de Lucio, I. «La I+D en España a través de publicaciones y patentes». En: Radiografía de la investigación pública en 
España (Jesús Sebastián y Emilio Muñoz, editores). Madrid: Biblioteca Nueva, 2006.

Cicyt. Plan Nacional de Investigación Científica, Desarrollo e Innovación Tecnológica 2000-2003. Madrid: Comisión Interministerial de Ciencia y Tecnología, 2000

Cicyt. Plan Nacional de Investigación Científica, Desarrollo e Innovación Tecnológica 2004-2007. Madrid: Comisión Interministerial de Ciencia y Tecnología, 2003, Isbn 8474749913.

Coronado, D.; Acosta, M. «The effects of scientific regional opportunities in science-technology flows: evidence from scientific literature in firms patent data». En: The annals of regional science, 2005, September, v. 39 , n. 3 , pp. $495-522$.

Cotec. La innovación en las tecnologías de la información y las comunicaciones. Madrid: Fundación Cotec para la Innovación Tecnológica, 2000, Isbn 84-95336-12-X. Informes Cotec sobre el Sistema Español de Innovación.

Cotec. Tecnología e innovación en España. Madrid: Fundación Cotec para la Innovación Tecnológica, 2001-2003, Isbn 84-95336-18-9, 84-95336-24-3, 84-95336-34-0.

Cuadrado-Roura, Juan R.; García-Tabuenca, Antonio. «ICT policies for SMEs and regional disparities. The Spanish case». En: Entrepreneurship \& regional development, 2004, January, v. 16, n. 1, pp. 55-75.

EITO. European Information Technology Observatory. Frankfurt, 2001.

European Commission (EC). Third European report on science \& technology indicators: towards a knowledge-based economy. Luxembourg: Office for Official Publications of the European Communities, 2003, Isbn 92-894-1795-1, Technical Report EUR [20025]-EN.

European Commission (EC). Science and technology in Europe. Statistical pocketbook. Data 1993-2003. Luxembourg: Office for Official Publications of the European Communities, 2005, Isbn 92-894-8729-1.

European Commission (EC). Monitoring industrial research: the 2005 EU industrial R\&D investment scoreboard. Luxembourg: Office for Official Publications of the European Communities, 2005, Isbn 92-79-00435-2, Technical Report EUR [21851] EN.

Fernández de Lucio, I.; Rojo, J.; Castro, E. Enfoques de políticas regionales de innovación en la Unión Europea. Madrid: Academia Europea de Ciencias y Artes, 2003 (Documento de trabajo).

Friedewald, M.; Kimpeler, S.; Hawkings, R.; Poel, M.; Lengrand, L.; Chattrie, I. Benchmarking national and regional policies in support of the competitiveness of the ICT sector in the EU. Interim report prepared for European Commission D4 under contract FIF [20030871], 2004.

Fundación AUNA. E-España 2005: informe anual sobre el desarrollo de la Sociedad de la Información en España. Madrid, 2005.

Gaptel. Productividad, crecimiento económico y TIC, Observatorio Español de Telecomunicaciones y de la Sociedad de la Información, Madrid: Grupo de Expertos en el Sector de las Telecomunicaciones, 2004.

Gómez, I.; Bordons, M.; Morillo, F.; Fernández, M. T. «Regionalisation of science and technology data in Spain». En: Research evaluation, 2005, August, v. 14, n. 2, pp. 137-148.

Gómez, I.; Sancho, R.; Bordons, M.; Fernández, M.T. «La I+D en España a través de publicaciones y patentes». En: Radiografía de la investigación pública en España (Jesús Sebastián y Emilio Muñoz, editores). Madrid: Biblioteca Nueva, 2006.

Guan, J.; Ma, N. «A comparative study of research performance in computer science». En: Scientometrics, 2004, January, v. 61, n. 3, pp. 339-359.

Hicks, D.; Brietzman, T.; Olivastro, D.; Hamilton, K. «The changing composition of innovative activity in the US - A portrait based on patent analysis». En: Research policy, 2001, April, v. 30, n. 4, pp. 681-703.

IKEI. Sector electrónico-informático y de telecomunicaciones. Vitoria-Gasteiz: Federación de Cajas de Ahorros Vasco-Navarras, 2002.

INE. Encuesta sobre innovación tecnológica en las empresas. 1998. Madrid: Instituto Nacional de Estadística, 1998.
Landabaso, M. «Las nuevas políticas regionales de promoción de la innovación en la Unión Europea». En: Revista de economía industrial, 2000, n. 335-336, V-VI, pp. 51-66.

Navarro, M. «La cooperación para la innovación en la empresa española desde una perspectiva internacional comparada». En: Economía industrial, 2002, n. 346, IV.

Mityc. Plan Avanza: plan 2006-2010 para el desarrollo de la sociedad de la información y de convergencia con Europa y entre comunidades autónomas y ciudades autónomas (Ingenio 2010). Madrid: Secretaría de Estado de Telecomunicaciones y para la Sociedad de la Información, 2005.

http://www.planavanza.es/documentacion.html

NSB. Science \& engineering indicators. Arlington VA, USA: National Science Foundation, 2002.

OECD. Measuring the ICT sector. Paris: Organisation for Economic Co-operation and Development, 2000.

OECD. Measuring the information economy. Paris: Organisation for Economic Co-operation and Development, 2002.

http://www.gdpglobal.co.uk/dbimgs/ICToecd.pdf

$O E C D$. Main science \& technology indicators/2. Paris: Organisation for Economic Co-operation and Development, 2003a.

$O E C D$. Science, technology and industry scoreboard. Paris: Organisation for Economic Co-operation and Development, 2003b.

OECD. ICT and economic growth: evidence from OECD countries, industries and firms. Paris: Organisation for Economic Co-operation and Development, 2003c.

$O E C D$. The economic impact of ICT: measurement, evidence and implications. Paris: Organisation for Economic Co-operation and Development, 2004a.

OECD. Information Technology Outlook. Paris: Organisation for Economic Co-operation and Development, 2004b.

OECD. Compendium of patent statistics. Paris: Organisation for Economic Co-operation and Development, 2004c.

OSIN. La sociedad de la información y las telecomunicaciones en Navarra. Navarra: Observatorio de la Sociedad de la Información en Navarra, 2003.

Pulido, Antonio. La innovación en el siglo XXI. Madrid, Ceprede (Centro de Predicción Económica), 2005.

Rojo, R.; Gómez, I. «Analysis of the Spanish scientific and technological output in the ICT sector». En: Scientometrics, 2006, December, v. 66, n. 1, pp. 101-121

Tsay, M-Y.; Ma, S-S. «The nature and relationship between the productivity of journals and their citations in semiconductor literature». En: Scientometrics, 2003, January, v. 56, n. 2, pp. 201-222.

Tijssen, R. J. W.; Van Wijk, E. «The global science base of information and communication technologies: bibliometric analysis of ICT research papers». En: Scientometrics, 1998, May, v. 42, n. 1, pp. 41-60.

Tijssen, R. J. W.; Van Wijk, E. «In search of the European paradox: an international comparison of Europe's scientific performance and knowledge flows in information and communication technologies research». En: Research policy, 1999, June, v. 28, n. 5, pp. 519-543.

$W I P O$. «The international patent classification ( $7^{\text {th }}$ edition)». Geneva: World Intellectual Property Organization, 2000.

Zitt, M.; Ramanana-Rahary, S.; Bassecoulard, E.; Laville, F. «Potential science-technology spillovers in regions: an insight on geographic co-location of knowledge activities in the EU». En: Scientometrics, 2003 January, v. 57, n. 2, pp. 295-320.

Raquel Rojo, Isabel Gómez, Cindoc-CSIC, Madrid. rrojo@cindoc.csic.es igomez@cindoc.csic.es 